Pacific Journal of Mathematic 


\title{
THE NUMBER OF FARTHEST POINTS
}

\author{
T. S. Motzkin, E. G. Straus, ani) F. A. Valentine
}

1. Introduction. Consider a set $S$ in a metric space $E$. For each point $x \in E$, let $y(x)$ denote a point of $S$ which has maximum distance from $x$, and let $Y(x)$ be the set of all $y(x)$ with that property. It is our purpose here to study sets $S$ for which certain restrictions are placed on the number of points in $Y(x)$. In $\S 2$ we analyze those sets $S$ in the Minkowski plane for which $Y(x)$ has exactly one element for each $x \in S$. In $\S 3$ we characterize those sets in the Euclidean plane $E_{2}$ for which $Y(x)$ has at least two elements for each $x \in S$.

In order to achieve these ends we first establish some introductory results which hold in rather general spaces.

DEFINITION 1 . Let $S$ be a set in a metric space. If $S$ is contained in a sphere of radius $r$, then its $r$-convex hull is the intersection of all closed spheres of radius $r$ which contain $S$.

A set $S$ is $r$-convex if it coincides with its $r-$ convex hull [2, p. 128].

Lemma 1. Let $S$ be a set of diameter $d$ in a linear metric space. Then for each $x \in S$ the set $Y(x)$ lies in the boundary of the $d$-convex hull of $S$.

Proof. If $Y(x) \neq 0$, choose any point $y(x)$. Then $S$ is contained in a sphere with center at $x$ and with radius $d(x, y)$, where $d(x, y)$ denotes the distance from $x$ to $y$. Since for $x \in S$ we have $d(x, y) \leq d$, there exists a point $z$ on the ray $\overrightarrow{y x}$ such that the sphere with center $z$ and with radius $d=d(z, y)$ contains $S$. The point $y$ is thus clearly on the boundary of the $d$-convex hull.

Note. By virtue of Lemma 1, all results for compact $S$ below will hold under the less restrictive assumption that $S$ contain the intersection of its closure with the boundary of its $d$-convex hull.

Corollary 1. Let $S$ be a set in a linear metric space. Then for each $x$ the set $Y(x)$ is contained in the boundary of the convex hull of $S$.

Received March 1, 1952. This work was performed on a National Bureau of Standards contract with the University of California, Los Angeles, and was sponsored (in part) by the Office of Scientific Research, U.S.A.F.

Pacific J. Math. 3 (1953), 221-232 
This is an immediate consequence of the fact that $S$ is contained in the sphere with center $x$ and radius $d(x, y(x))$, provided $Y(x) \neq 0$.

Lemma 2. Suppose $S$ is a set in a linear metric space, and let $T$ be a set such that $Y(x) \neq 0$ for each $x \in T$. Then $d(x, y(x))$ is a continuous function of $x$ on $T$.

$$
\begin{aligned}
& \text { Proof. Since }|d(x, z)-d(u, z)| \leqq d(x, u) \text {, and since } \\
& \qquad \max _{z \in S} d(x, z)-\max _{z \in S} d(u, z)|=| d(x, y(x))-d(u, y(u)) \mid,
\end{aligned}
$$

we have $|d(x, y(x))-d(u, y(u))|<\epsilon$ if $d(x, u)<\epsilon$.

LeMma 3. Let $S$ be a compact set in a linear metric space. If $x_{i} \rightarrow x$, then all limit points of the sequence $\left\{y\left(x_{i}\right)\right\}$ lie in $Y(x)$.

Proof. Let $y_{i}=y\left(x_{i}\right)$ be a sequence of points. Let $y$ be a limit point of the sequence $\left\{y_{i}\right\}$. Then the continuity of $d(x, y(x))$ implies that $d(x, y) \geq d(x, q)$ for all $q \in S$. Hence we have $y^{\prime} \in Y(x)$.

LEMMA 4. Let $S$ be a compact set in a linear metric space, and suppose $y(x)$ is single-valued on a set T. Then $y(x)$ is a continuous mapping of $T$ into $S$.

Proof. Since $y(x)$ is single - valued, Lemma 3 implies that if $x_{i} \longrightarrow x$, then $y\left(x_{i}\right) \rightarrow y(x)$.

2. Sets in $M_{2}$ on which $y(x)$ is single - valued. Let $M_{2}$ be a two - dimensional Minkowski space [2, p. 23]. We restrict our attention here to connected sets $S$ in $M_{2}$. (See $\$ 4$ for remarks about disconnected sets.)

THЕОRЕM 1. Let $S$ be a continuum (compact connected set) in $M_{2}$. If $y(x)$ is single-valued on $S$, then the set sum

$$
\sum_{x \in S} Y(x)
$$

is the entire boundary $B$ of the convex hull of $S$; and this convex hull is $d$ convex, where $d$ is the diameter of $S$.

Proof. According to Corollary 1, we have

$$
\sum_{x \in S} Y(x) \subseteq B
$$


By Lemma 4, the mapping $y(x)$ yields a continuous mapping of $S$ into $B$. Now the only connected sets in a simple closed curve are: (1) a point, (2) a simple arc, (3) the whole closed curve. For cases (1) and (2), let

$$
A \equiv \sum_{x \in S} Y(x)
$$

then the mapping $y(x)$ of $A$ into itself must have a fixed point $x_{0}=y\left(x_{0}\right)$, so that $\left\{x_{0}\right\}=Y\left(x_{0}\right)=S$, in which case the theorem is trivial. Thus $A=B$ in all three cases. Moreover, since by Lemma 1 the set $A=B$ lies in the boundary of of the $d$-convex hull of $S$, the boundary of the $d$-convex hull must coincide with B.

Since there is no continuous mapping without fixed points of a closed twocell into itself, Lemma 2 and Theorem 1 imply that, for single-valued $y(x)$, the connected bounded set $S$ must contain the entire boundary of its convex hull, but not all of the interior of that hull (unless $S$ consists of a single point). It may suffice, in some cases, to delete one single point from the interior of a convex set; for instance, in the case of a circular disc in $E_{2}$, the deletion of the center makes $y(x)$ single-valued throughout.

In the remaining theorems and lemmas we restrict our attention to sets in $E_{2}$.

Definition 2. By a normal to a convex curve $C$ at a point $x \in C$ we mean a line perpendicular to a line of support to $C$ at $x$.

Notation. We designate a line of support at $x$ by $L(x)$, and the corresponding normal by $N(x)$. Further, for a point $y \in S$, we let $x(y)$ be a point in $S$ such that $y=y(x)$, and let $X(y)$ be the set of all $x(y)$.

THEOREM 2. Suppose $S$ is the boundary of a compact convex set in $E_{2}$, and suppose $y(x)$ is single-valued on $S$. Then:

(1) The set $X(y)$ consists of all points of intersection of the normals to $S$ at $y$ with $S-y$. If $S$ has a tangent at $y$, then $x(y)$ is single-valued and continuous at $y$.

(2) The mapping $x(y)$ is monotonic; that is, the order of $x\left(y_{1}\right), x\left(y_{2}\right)$, $x\left(y_{3}\right)$ on $S$ has the same sense as that of $y_{1}, y_{2}, y_{3}$.

Proof. (1) If $x=x(y)$, then the circle with center $x$ and radius $d(x, y)$ contains $S$. Hence the tangent to this circle at the point $y$ is also a line of support to $S$, and the radius lies in a normal to $S$ at $y$. 
Now, let $y_{i} \longrightarrow y, y_{i} \in S$, and choose $x_{i}=x\left(y_{i}\right)$. Then, due to the continuity of the mapping $y(x)$, each limit point of $\left\{x_{i}\right\}$ is in $X(y)$. Thus if $S$ has a tangent at a point $y$, then the mapping $x(y)$ is one-to-one and continuous at $y$.

To complete the proof of ( 1 ), suppose $S$ has a corner at $y$. Then the farthest points of intersection from $y$ of the normals at $y$ with $S$ fill out a closed subarc of $S$, which we denote by $S_{1}$; the end-points of $S_{1}$ we denote by $u_{l}$ and $u_{r}$. There exists a sequence $y_{i} \in S$ with $y_{i} \rightarrow y$ such that the normals to $S$ at the $y_{i}$ are unique and approach the left normal at $y$. Hence, by the above, $x\left(y_{i}\right)$ converges to $u_{l}$, and hence $u_{l} \in X(y)$. Similarly, $u_{r} \in X(y)$. The three lines determined by $u_{l}, u_{r}$, and $y$ divide the plane into seven closed sets, and the arc $S_{1}$ is contained in that unbounded one which has $u_{l} u_{r}$ as part of its boundary. We denote that set by $A$. Since each of the two circles with centers $u_{l}$ and $u_{r}$ which pass through $y$ contains $S$, it follows by the law of cosines that $y(u)=y$ for all $u \in A$. Hence $S_{1} \subseteq X(y)$. According to Theorem 1 , the curve $S$ contains no straight line-segment, and thus any normal to $S$ intersects $S$ in exactly two points. Hence the common part $\left(S-S_{1}\right) \cdot X(y)$ is the null set, so that $S_{1}=$ $X(y)$.

(2) The above facts, together with the fact that each $u \in S$ is contained in some $X(y)$, imply that the transformation $x(y)$ maps connected sets into connected sets, even though the mapping need not be single-valued and therefore not necessarily continuous. The single-valuedness of $y(x)$ implies that if $y_{1} \neq y_{2}$, then $X\left(y_{1}\right) \cdot X\left(y_{2}\right)=0$. If the transformation $x(y)$ failed to be monotonic, it would have a fixed point $y=x(y)$; but this is impossible unless $S$ is a single point. Hence condition (2) must hold.

Corollary 2. Suppose $C$ is the boundary of a compact convex set $S$. Let $\alpha \beta$ be a diameter of $C$, and let $N(\alpha, \beta)$ designate the common normal to $C$ through $\alpha$ and $\beta$. Then $y(x)$ is single-valued on $C$ if and only if for every pair of points $u, v \in C$ which lie on the same side of $N(\alpha, \beta)$, the normals $N(u)$ and $N(v)$ intersect at an interior point of $S$.

Proof. First observe that, for any compact convex set $S$ with $\alpha \beta$ as a diameter, if $x \cdot \alpha \beta=0$, then $x$ and $y(x)$ must lie on opposite sides of $N(\alpha, \beta)$.

To prove the necessity, observe that $\alpha$ and $\beta$ are involutory points in the sense that

$$
y(y(\alpha))=\alpha \text { and } y(y(\beta))=\beta
$$

Hence the necessity follows from the monotonicity of $y(x)$ as described in 
Theorem 2.

To prove the sufficiency, first choose $x \in C-(C \cdot \alpha \beta)$. Suppose $y(x)$ is not single-valued, and choose $u, v \in Y(x)$. As mentioned above, $y(x)$ and $x$ lie on opposite sides of $N(\alpha, \beta)$. A circle with center $x$ and radius $d(x, u)$ is tangent to $C$ at both $u$ and $v$, and the normals $N(u)$ and $N(v)$ intersect at $x$, which is not interior to $S$. Hence $y(x)$ is single-valued for $x \in C-(C \cdot \alpha \beta)$. By continuity it follows also that

$$
y(\alpha)=\beta, y(\beta)=\alpha \text {. }
$$

This completes the proof.

In the following we shall extend the generalized notions of curvature described by Bonnesen and Fenchel [2, pp. 143-144]. Choose a point $x \in C$, where $C$ is a closed convex curve together with a line of support $L(x)$. The circle tangent to $L(x)$ at $x$ and passing through a point $p \in C-x$ must have its center $z(p)$ on the normal $N(x)$ to $L(x)$ at $x$. Establish an order on $N(x)$ in terms of the distance from $x$, and let

$$
\left.\begin{array}{l}
E_{s}(x, \delta(x)) \equiv \sup _{p} z(p) \\
E_{l}(x, \delta(x)) \equiv \inf _{p} z(p)
\end{array}\right\} p \in \delta(x)-x,
$$

where $\delta(x)$ is an arc of $C$ containing $x$. We define four types of centers of curvature as follows:

$$
\begin{gathered}
E_{s}(x) \equiv E_{s}(x, C), \quad E_{l}(x)=E_{l}(x, C) \\
E_{o}(x) \equiv \lim _{\delta(x) \rightarrow x} E_{s}(x, \delta(x)), \quad E_{i}(x) \equiv \lim _{\delta(x) \rightarrow x} E_{l}(x, \delta(x)) .
\end{gathered}
$$

Clearly $E_{l}(x) \leq E_{i}(x) \leq E_{o}(x) \leq E_{s}(x)$ relative to $N(x)$

DEFINITION 3. The sets

$$
\sum E_{s}(x), \sum E_{o}(x), \sum E_{i}(x), \text { and } \sum E_{l}(x)
$$

( $x$ ranges over $C$ ) are respectively called the superior evolute, the outer evolute, the inner evolute, and the inferior evolute of $S$, and are denoted by $E_{s}, E_{o}, E_{i}, E_{l}$.

THEOREM 3. Suppose $C$ is the boundary of the compact convex set $S \subset E_{2}$. If $y(x)$ is single-valued on $C$, then the superior evolute, and hence all four 
evolutes, of $C$ must be contained in $S$.

Proof. Since $y(x)$ is single-valued for each point $x_{1} \in C$, the proof of Theorem 2 implies that for any normal $N\left(x_{1}\right)$, the set $N\left(x_{1}\right) \cdot\left(C-x_{1}\right)$ consists of a single point, denoted by $x^{\prime}$. Choose $p \in C-x_{1}$. Since

$$
d\left(x^{\prime}, p\right)<d\left(x^{\prime}, x_{1}\right)=d\left(x^{\prime}, y\left(x^{\prime}\right)\right),
$$

it is clear that the perpendicular bisector $B$ of the segment $x_{1} p$ intersects the segment $x_{1} x^{\prime}$. Hence

$$
B \cdot x_{1} x^{\prime}=z(p) \in S .
$$

THEOREM 4. Suppose the inner evolute of the boundary $C$ of the compact convex set $S$ is contained in $S-C$. Then $y(x)$ is single-valued on $C$.

Proof. Suppose there exists an $x \in C$ such that $y(x)$ is not single-valued. Choose $u, v \in Y(x)$. The circle with center $x$ and radius $d(x, u)$ contains $S$ and is tangent to $C$ at $u$ and $v$. Hence the arc $u v$ of $C-x$ contains a point $w$ of minimal distance from $x$. The circle with center $x$ and radius $d(x, w)$ is tangent to $C$ at $w$, while a neighboring arc of $w$ on $C$ lies outside or on that circle. Hence $C$ has a unique normal at $w$ and $E_{i}(w) \geq x$, so that $E_{i}(w)$ is on or outside $C$.

Theorems 3 and 4 do not determine the single-valuedness of $y(x)$ on $S$ if $E_{i}$, $E_{o}$, and $E_{s}$ lie in $S$ and contain points of $C$. This situation can be described as follows:

THЕОRЕM 5. Let $S$ be a compact convex set with boundary $C$ such that $E_{s}$ (and hence each of the evolutes) of $C$ lies in S. Then $y(x)$ fails to be singlevalued on $C$ if and only if there exists a point $x \in C$ which lies on $E_{i}, E_{o}$, and $E_{s}$, and which is the center of a circular arc contained in $C^{\mathbf{1}}$

Proof. To prove sufficiency, suppose there exists a point $x \in C$ which is the center of a circular arc $C_{1} \subset C$, and suppose $y(x)$ is single-valued on $C$. Then according to Theorem 2 the single-valuedness of $y(x)$ implies $x \in X(y)$ for each $y \in C_{1}$. Hence $C_{1} \subseteq Y(x)$, a contradiction.

To prove necessity, assume $y(x)$ is not single-valued on $C$. Choose $u$, $v \in Y(x)$, and let $w$ be a nearest point to $x$ of the arc $C_{1}$ of $C-x$ joining $u$ and $v$. In the proof of Theoram 4 we saw that $E_{i}(w) \geq x$; but since the evolutes are

\footnotetext{
${ }^{1}$ By "center of a circular arc" we mean the center of the circle to which the arc belongs.
} 
in $S$, we have

$$
E_{i}(w)=E_{o}(w)=E_{s}(w)=x
$$

(Since $E_{s}$ is bounded, $C$ can contain no straight line segments.) Hence the circle with center $x$ and radius $d(x, w)$ contains $S$. Thus $d(x, w) \geq d(x, u)$. From the definition of $w$ it now follows that $d(x, z)=d(x, u)$ for each $z \in C_{1}$. Hence $C_{1}$ Hence $C_{1}$ is circular arc in $C$ with center at $x$.

As seen earlier, if $S$ is a simply connected set containing at least two points, then $y(x)$ is not single-valued on $S$. The situation is described more fully in the following theorem.

THEOREM 6. Let $S$ be a compact convex set in $E_{2}$ with boundary $C$. Then $y(z)$ is single-valued if $z \neq E_{s}(x)$ for all $x \in C$; and $y(z)$ is not singlevalued if $z=E_{s}(x), z \neq E_{o}(x)$ for some $x \in C$.

Proof. Assume $y(z)$ is not single-valued; then there exist distinct points $u \in Y(z), v \in Y(z)$, and the circle with center $z$ and radius $d(z, u)$ contains $S$ and is tangent to $C$ at $u$ and $v$. Hence $E_{s}(u)=E_{s}(v)=z$.

Now suppose there exists an $x \in C$ such that $z=E_{s}(x), z \neq E_{o}(x)$. Then, since $C$ is compact, there exists a point $u \neq x, u \in C$, such that

$$
d(z, u)=d(z, x)=d(z, y(z)) \text {. }
$$

Hence $u \in Y(z), x \in Y(z)$. Thus Theorem 6 is proved.

A few remarks about the four evolutes may be desirable at this point. The inferior and superior centers of curvature, $E_{l}(x)$ and $E_{s}(x)$, are determined by properties in the large. In fact, $E_{l}$ contains the set of centers of those circles which are in $S$ and which are tangent to $C$ at not less than two points. Similarly $E_{s}$ contains the sets of centers of those circles which contain $C$ and which are tangent to $C$ at not less than two points.

Since a convex curve $C$ has curvature almost everywhere, we have $E_{i}(x)=$ $E_{o}(x)$ for almost all $x \in C$. Let us define

$$
E \equiv \sum E_{i}(x) E_{o}(x),
$$

( $x$ ranges over $C$ ), where, as usual, $E_{i}(x) E_{o}(x)$ denotes a closed segment. The number of normals to $C$ through a point $x \in E_{2}$, as a function of $x$, is the same in each component of the complement of $E$. In the case where $S$ is a compact convex set for which $E$ is bounded, there are exactly two normals to $C$ through each point $x$ in the unbounded component of the complement of $E$ (the 
lines joining $y$ to the nearest and farthest points on $C$ ). However, from each point $y \notin E$ on $E_{l}\left(E_{s}\right)$ there are at least four normals to $C$. [According to Theorem 6, there are at least two normals to the two or more points of tangency $u, v$ of the inscribed (circumscribed) circle with center at $y$. In addition, there are lines joining $y$ to nearest (farthest) points on each of the two arcs of $C$ joining $u$ and $\left.v_{.}\right]$Thus $E_{l}$ and $E_{s}$ do not intersect the unbounded component of $\bar{E}$. These statements imply the following:

THEOREM 7. Let $C$ be the boundary of a compact convex set $S \subset E_{2}$. Then $E_{s} \subset S$ if and only if $E_{o} \subset S$. Also $E_{s} \subset S-C$ if and only if $E_{o} \subset S-C$.

AN EXAMPLE. Consider the family of ellipses $C(e)$,

$$
b^{2} x_{1}{ }^{2}+a^{2} x_{2}^{2}=a^{2} b^{2}, a \geqq b \text {. }
$$

If the eccentricity $e$ satisfies the condition $e \leq \sqrt{2} / 2$, then $y(x)$ is singlevalued on $C(e)$. If $e>\sqrt{2} / 2$, then $y(x)$ is not single-valued at $x=(0, \pm b)$. In each case the inner and outer evolutes coincide; they form the familiar astroid with cusps at

$$
\xi=\left(a_{1}, 0\right), \eta=\left(-a_{1}, 0\right), \tau=\left(0, b_{1}\right) \text { and } \rho=\left(0,-b_{1}\right),
$$

where $a_{1}<a$, and $b_{1}<b$ for $e<\sqrt{2} / 2$ while $b_{1}>b$ for $e>\sqrt{2} / 2$. The superior evolute $E_{s}$ is the closed line-segment $\rho \tau$, and $E_{l}$ is the closed line-segment $\xi \eta$. If $e \neq 0$, then $y(x)$ is single-valued on the complement of the open segment $\rho \tau-\rho-\tau$.

\section{Sets on which $Y(x)$ contains at least two points.}

THEOREM 8. Let $S \subset E_{2}$ be a compact set of diameter $d_{,}$and let $D$ denote the set of end-points of diameters of $S$. If $Y(x)$ has at least two elements for each $x \in D$, then $Y(x)$ consists of exactly two points for $x \in D$, and $D$ contains a finite number of points. The $d$-convex hull of $S$ coincides with the dconvex hull of $D$. [Since the latter is a Reuleaux polygon ( see below), $D$ must contain an odd number of points.]

Proof. Let $\Sigma \equiv\{C(x)\}$ be the family of circular boundaries $C(x)$ with centers $x \in D$ and with radii $d$. Let $x \in D$; then

$$
Y(x)=C(x) \cdot D \text {. }
$$

Since

$$
\operatorname{diam} Y(x) \leq \operatorname{diam} S=d,
$$


there exists a smallest arc $A$ of $C(x)$ which contains $Y(x)$, and which has a length not exceeding $\pi d / 6$. Let $x_{1}$ and $x_{2}$ be the end-points of $A$. If a circle $C\left(x^{\prime}\right) \in \Sigma$ were to intersect $A-x_{1}-x_{2}$, then $C\left(x^{\prime}\right)$ would separate $x_{1}$ and $x_{2}$ since

$$
\text { length } A \leqq \pi d / 6 \text {. }
$$

But this contradicts the fact that $S \subset C\left(x^{\prime}\right)$. For any $x \in D$, we have $z=y(x)$ if and only if $x=y(z)$. Hence every $x \in D$ is a point of intersection of at least two circles of $\Sigma$. These facts imply that $Y(x) \equiv\left\{x_{1}, x_{2}\right\}$.

Define

$$
H \equiv \prod_{x \in D} K(x)
$$

where $K(x)$ is the closed circular disk with center $x$ and with radius $d$. Then each $x \in D$ lies in the interior of all $K(x) \subset H$ except $K\left(x_{1}\right)$ and $K\left(x_{2}\right)$, where $Y(x)=\left\{x_{1}, x_{2}\right\}$. Hence $x$ is a corner-point of the boundary of $H$. As above, let $A_{1}$ and $A_{2}$ be the smallest arcs of $C\left(x_{1}\right)$ and $C\left(x_{2}\right)$ containing $Y\left(x_{1}\right)$ and $Y\left(x_{2}\right)$, respectively. We have shown that $A_{1} \cdot A_{2}=\{x\}$; and $A_{1}$ and $A_{2}$ are in the boundary of $H$. Thus $x$ is an isolated corner of the boundary of $H$. Hence $D$ contains a finite number of points, and by definition the boundary of $H$ is the boundary of the $d$-convex hull of $D$. It is clearly a Reuleaux polygon, that is, a convex circular polygon whose arcs have radii $d$, and whose vertices are the centers of these arcs [2, pp. $130-131]$.

Finally, each of the circles in $\Sigma$ contains $S$, and hence $S \subset H$.

CoROLLARY 3. Let $S$ be a set satisfying the conditions of Theorem 8. Then $Y(x) \subseteq D$ for each $x \in S$.

This is an immediate consequence of the fact that $D$ consists of the vertices of $H$.

THEOREM 9. Let $S \subset E_{2}$ be a compact set such that $Y(x)$ has at least two elements for each $x \in S$. Then $S$ lies in the union of a finite number of linesegments. Moreover, if $Y(x)$ has exactly two elements for each $x \in S$, then $S$ cannot be connected.

Proof. Since $Y(x) \subseteq D$ for each $x \in S$, the fact that $Y(x)$ has a least two elements implies that $x$ lies on the perpendicular bisector of the line joining two elements of $D$. Thus $S$ is a subset of the set obtained by taking the union of the intersections of these perpendicular bisectors with $H$. 
Since the set $H$ has at least three corners $x_{1}, x_{2}$ and $x_{3}$, let $S_{i}(i=1,3)$ consist of those $x \in S$ such that $\left\{x_{i}, x_{2}\right\} \subseteq Y(x)$. Each set $S_{i}$ is nonempty since $S_{i}$ contains the center of the smaller arc of $H$ joining $x_{i}$ and $x_{2}$. From the continuity of $d(x, y(x))$, it follows that $S_{i}$ is closed. Hence if $S$ is connected, then $S_{1} \cdot S_{2} \neq 0$ ( since $S$ is compact), and thus there exists an $x^{\prime} \in S$ such that $Y\left(x^{\prime}\right) \supseteq\left\{x_{1}, x_{2}, x_{3}\right\}$. This establishes the theorem.

We also obtain the following result due to Bing [1].

Corollary 4. Let $S$ be a bounded set in $E_{2}$ containing at least two points, and having the property that with every two points $x \in S, y \in S$ there exists a $z \in S$ such that the triangle $x y z$ is equilateral. Then $S$ is the set of vertices of an equilateral triangle.

Proof. The closure $\bar{S}$ of $S$ must also satisfy the hypothesis stated. Consider the set $D$ of Theorem 8 relative to $\bar{S}$. If $x \in D$, and $\{y, z\} \subseteq Y(x)$, then $d(y, z)=$ $d$, so that $x, y, z$ form the vertices of a Reuleaux polygon, and therefore by Theorem 8 we have $D=\{x, y, z\}$. Now let $u$ be the centroid of the triangle $x, y$, $z$. By Theorem $9, S$ is contained in the segments $x u, y u$, and $z u$. Suppose $v \in$ $(S \cdot x u-x)$; then $Y(v)=\{y, z\}$. But $v, y, z$ is not equilateral; hence $S \cdot x u=$ $x$. Similarly, $S \cdot y u=y, S \cdot z u=z$. Consequently, $S=\{x, y, z\}$.

4. Remarks and problems. Several questions are raised by our theorems.

(1) If we try to characterize disconnected sets in $E_{2}$ for which $y(x)$ is single-valued, we see that this condition is not very restrictive. In fact, given any set $S$ which contains at least one point of the boundary of its $r$-convex hull $H$ for some radius $r$, we can adjoin a single point $z$ to $S$, such that $z$ lies on an interior normal to $H$ at a point of $H \cdot S$, and such that $y(x)$ relative to $S+\{z\}$ is single-valued on $S+\{z\}$.

(2) The characterization of connected sets $S$ in $E_{n}(n>2)$ for which $y(x)$ is single-valued on $S$ offers considerable difficulties. The mapping $y(x)$ still yields a continuous map of $S$ into the boundary of its convex hull, but it need no longer be an onto mapping. For example, the torus, both the solid and its surface, will have single-valued $y(x)$ for suitable ratios of the two radii. The argument that a nontrivial compact $S$ which contains no indecomposable continua cannot be simply - connected holds, however, regardless of dimension, since every continuous mapping of such a simply-connected set $S$ into itself has fixed points [4].

(3) The generalization of the discussion of multivalued $y(x)$ suggests the 
following problem: Let $S$ be a compact set in $E_{n}$ such that $Y(x)$ has at least $k$ elements for $x \in S$. Does it follow that $S$ lies in the union of a finite number of $(n-k+1)$-dimensional planes? (Note that in the case $k=1$ this is no restriction, while for $k>n+1$ there would be no sets $S$.) Are there any sets for which $k=n+1 ?$

It seems likely that this generalization is false, since the argument which proved the finiteness of the set $D$ in Theorem 8 fails for $n>2$.

In the case $k \geq n$, all points of $D$ are vertices of their $d$-conves hull. Thus in this case $D$ must surely be denumerable.

(4) Is it possible to generalize Corollary 4, as follows:

If the bounded set $S$ in $E_{n}$ contains at least two points; and if, for some $k \geq 2$, with every two points $x, y \in S$ there are $k-1$ points in $S$ which together with $x, y$ form the vertices of a regular $k$-simplex, does it follow that $S$ is the set of vertices of a regular $l$-simplex, where $k \leq l \leq n$ ?

(5) Another question raised by Corollary 4 is the following:

What are the sets (bounded sets, compact sets) $S$ in $E_{2}$ which have the property that with every $x, y \in S$ there is a $z \in S$ such that $x y z$ is an isosceles triangle with vertex $z$ and prescribed verticle angle $\alpha$ ?

For $\alpha<\pi / 3$, a nontrivial set with the stated property obviously cannot be bounded. For $\alpha=\pi / 3$, the question for the bounded case is answered by Corollary 4. For $\alpha>\pi / 3$, there is a considerable variety of bounded sets, although none of them can be finite. In fact, for $\alpha>\pi / 3$ every $S$ must be dense in itself; and thus, if closed, it must be perfect. The case $\alpha=\pi$ has been discussed by J. W. Green and W. Gustin [3]; for closed sets $S$, this case characterizes convexity.

An easy argument shows that for compact $S$, and $\pi / 3<\alpha \leq \pi / 2$, the entire line-segment joining two farthest points of $S$ must be contained in $S$.

It may also be worth remarking that if $S$ has the foregoing property for an angle $\alpha$, then its complement has the same property for the angle $\pi-\alpha$. Thus the case $\alpha=\pi / 2$ is especially noteworthy, since in this case the class of all $S$ with the stated property is closed under the operation of taking complements.

(6) Finally, one should compare the theorems about $Y(x)$ with those for $M(x)$, where $M(x)$ denotes the subset of $S$ whose points have minimum distance from $x$. In particular the theorem of Motzkin [6, 7] (see also Jessen [5]) states that a closed set $S$ is convex if and only if $M(x)$ is a single point for all $x$. This theorem does not correspond to any of the results on $Y(x)$ in $\$ 1$. In fact, the 
analogous assumption, concerning a (not necessarily closed) set $S$ in $E_{n}$, that $y(x)$ be single - valued for all $x$, is satisfied if and only if $S$ consists of a single point.

\section{REFERENCES}

1. R. H. Bing, On equilateral distance, Amer. Math. Monthly 58 (1951), 380-383.

2. T. Bonnesen and $W$. Fenchel, Theorie der konvexen Körper, Julius Springer, Berlin, 1934; reprinted by Chelsea, N.Y., 1948.

3. J. W. Green and W. Gustin, Quasiconvex sets, Canadian J. Math. 2 (1950), 489507.

4. O. H. Hamilton, Fixed points under transformations of continua which are not connected im kleinen, Trans. Amer. Math. Soc. 44 (1938), 719-724.

5. B. Jessen, Two theorems on convex point sets (Danish), Mat. Tidsskr. B (1940), 66-70.

6. T. S. Motzkin, Sur quelques propriétés caractéristiques des ensembles convexes, Rendiconti della Reale Academia dei Lincei, Classe di Scienze Fisiche, Matematiche e Naturali, 21 (1935), 562-567.

7. - Sur quelques propriétés caractéristiques des ensembles bornés non convexes, Rendiconti della Reale Academia dei Lincei, Classe di Scienze Fisiche, Matematiche e Naturali, 21 (1935), 773-779.

University of California, Los Angeles 


\section{PACIFIC JOURNAL OF MATHEMATICS}

\section{EDITORS}

\author{
R. M. Foeinson \\ University of California \\ Berkeley 4, California \\ E. HEWITt \\ University of Washington \\ Seattle 5, Washington
}

R. P. DILWOR TH

California Institute of Technology

Pasadena 4, California

E. F. BECKENBACH

University of California

Los Angeles 24, California

\section{ASSOCIATE EDITORS}

$\begin{array}{llll}\text { H. BUSEMANN } & \text { P. R. HALMOS } & \text { BøRGE JESSEN } & \text { J. J. STOKER } \\ \text { HERBERT FFDERER } & \text { HEINZ HOPF } & \text { PAUL LÉVY } & \text { E. G. STR AUS } \\ \text { MARSHALL IIALI } & \text { R. D. JAMES } & \text { GEORGE PÓLYA } & \text { KỎSAKU YOSIDA }\end{array}$

\section{SPONSORS}

UNIVERSITY OF BRITISH COLUMBIA CAIIFORNIA INSTITUTE OF TECHNOLOGY UNIVERSITY OF CALIFORNIA, BERKELEY UNIVERSITY OF CAILIFORNIA, DAVIS UNIVERSITY OF CALIFORNIA, LOS ANGELES UNIVERSITY OF CALIFORNIA, SANTA BARBARA UNIVERSITY OF NEVADA OREGON STATE COLLEGE UNIVERSITY OF OREGON
UNIVERSITY OF SOUTHERN CALIFORNIA STANFORD RESEARCH INSTITUTE STANFORD UNIVERSITY WASHINGTON STATE COLLEGE UNIVERSITY OF WASHINGTON

AMERICAN MATHEMATICAL SOCIETY NATIONAL BUREAU OF STANDARDS, INSTITUTE FOR NUMERICAL ANALYSIS

$$
\begin{gathered}
\text { Vari-Type Composition by } \\
\text { Elaine Barth } \\
\text { Delores Wierman } \\
\text { With the cooperation of } \\
\text { E. F. Beckenbach } \\
\text { E. G. Straus }
\end{gathered}
$$

Printed in the United States of America by Edwards Brothers, Inc., Ann Arbor, Michigan 


\section{Pacific Journal of Mathematics}

\section{Vol. 3, No. 1 \\ March, 1953}

Herbert Busemann, Volume in terms of concurrent cross-sections ......... 1

L. Carlitz, Some special equations in a finite field ................. 13

Homer V. Craig and Billie Braden Townsend, On certain metric

extensors ....................................... 25

Philip J. Davis and Henry Pollak, Linear functionals and analytic

continuation problems ............................. 47

Jacob C. E. Dekker, The constructivity of maximal dual ideals in certain

Boolean algebras ................................. 73

Harley M. Flanders, The norm function of an algebraic field extension .... 103

Marshall Hall, Subgroups of free products . . . . . . . . . . . . . . . . . 115

Israel (Yitzchak) Nathan Herstein, Finite multiplicative subgroups in

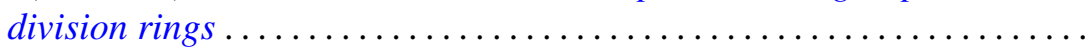

Joseph Lawson Hodges, Jr. and Murray Rosenblatt, Recurrence-time moments in random walks ............................ 127

Alfred Horn, The normal completion of a subset of a complete lattice and lattices of continuous functions ........................ 137

Fulton Koehler, Estimates for the errors in the Rayleigh-Ritz method...... 153

M. H. Martin, The Monge-Ampère partial differential equation

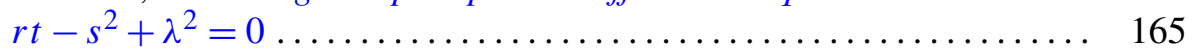

John E. Maxfield, Normal k-tuples ......................... 189

Jack E. McLaughlin, Structured theorems for relatively complemented lattices............................................ 197

William H. Mills, A system of quadratic Diophantine equations ......... 209

T. S. Motzkin, Ernst Gabor Straus and F. A. Valentine, The number of farthest points ................................. 221

G. Power, Forces on the boundary of a dielectric ............... 233

Ralph Gordon Selfridge, Approximations with least maximum error....... 247 